\title{
Metalloproteinases in Inflammatory Bowel Diseases
}

This article was published in the following Dove Press journal:

Journal of Inflammation Research

\author{
Martin Marônek (D) ${ }^{1} *$ \\ Irene Marafini ${ }^{2}$,* \\ Roman Gardlík (D) \\ René Link ${ }^{3}$ \\ Edoardo Troncone ${ }^{2}$ \\ Giovanni Monteleone ${ }^{2}$ \\ 'Institute of Molecular Biomedicine, \\ Faculty of Medicine, Comenius University \\ in Bratislava, Bratislava, 8I I08, Slovakia; \\ ${ }^{2}$ Department of Systems Medicine, \\ University of Rome "Tor Vergata", Rome, \\ 00133, Italy; ${ }^{3}$ Institute of Experimental \\ Medicine, Faculty of Medicine, University \\ of Pavol Jozef Šafárik, Košice, 040 II, \\ Slovakia
}

*These authors contributed equally to this work
Correspondence: Giovanni Monteleone Dipartimento di Medicina dei Sistemi, Università di Roma "Tor Vergata", Via Montpellier I, Rome, 00133, Italy

Tel +390620903702

$\mathrm{Fax}+390672596391$

Email Gi.Monteleone@Med.uniroma2.it
Abstract: Inflammatory bowel diseases (IBD) are chronic inflammatory diseases of the gastrointestinal tract, encompassing two main disorders: Crohn's disease (CD) and ulcerative colitis (UC). In both these pathologies, excessive and local immune response against luminal antigens promotes a pathological process leading to various degrees of gut damage. Matrix metalloproteinases (MMPs) are a family of neutral proteases with the ability to degrade all components of extracellular matrix. In physiological conditions, MMPs are produced at very low level and generally in the latent form and are involved in the normal tissue turnover. Their function is inhibited by tissue inhibitors of metalloproteinases (TIMPs). However, in inflamed tissue of IBD patients, MMPs are produced in excess and/or the activity of TIMPs is not sufficient to block MMPs, thereby making a major contribution to the IBD-related mucosal degradation. In this review, we summarize the available evidence on the expression and role of MMPs in IBD.

Keywords: tissue inhibitor of metalloproteinases, Crohn's disease, ulcerative colitis, intestinal inflammation

\section{Introduction}

Inflammatory bowel diseases (IBD) are chronic inflammatory diseases of the gastrointestinal tract, encompassing two main clinical forms: Crohn's disease (CD) and ulcerative colitis (UC). IBD are thought to be caused by interaction of genetic and environment factors, ${ }^{1}$ which eventually promotes an abnormal immune reaction direct against components of the luminal flora. ${ }^{2}$ It is estimated that in Europe alone there are around 2.6 million people suffering from IBD with another approximately 1.2 million in North America. ${ }^{3}$ Around $25 \%$ of the patients are diagnosed before the age of 18 years. ${ }^{4}$ A curative therapy does not exist and the aim of current treatments is to suppress intestinal inflammation. Despite the therapeutic armamentarium has been expanded in recent years, some IBD patients do not respond to specific drugs or can develop drug-related serious adverse events, thus suggesting the necessity of alternative therapeutic agents. ${ }^{5}$

In IBD, the pathological process is associated with an extensive mucosal degradation and tissue remodeling, which ultimately favor the development of ulcers, fistulae and strictures. Although the pathogenesis of such lesions is not yet fully understood, a large body of evidence supports the involvement of multiple proteases produced within the inflammatory microenvironment in these processes. One example of such proteases is given by matrix metalloproteinases (MMPs), a group of zinc-dependent neutral peptidases capable of degrading all components of the extra-cellular matrix 
(ECM). ${ }^{6}$ MMPs are mostly secreted as latent, inactive zymogens by multiple cell types, including myofibroblasts, T cells, macrophages, monocytes, neutrophils, and epithelial cells. Conversion into active enzyme usually occurs in the pericellular or extracellular space. According to their primary substrate, MMPs can be divided into various subclasses: collagenases (MMP-1, MMP-8, MMP-13 and MMP-18), gelatinases (MMP-2 and MMP-9), stromelysins (MMP-3, MMP-7, MMP-10 and MMP-11), elastase (MMP-12), membrane types (MMP-14, MMP-15, MMP-16, MMP-17, MMP-24 and MMP-25) and others (MMP-19, MMP-20, MMP-23, MMP-26, MMP-27 and MMP-28). ${ }^{7}$ (Table 1). MMPs act in concert to create a cascade of activation; following activation, one MMP can trigger the conversion of other MMP zymogens to their active forms (Figure 1A), thereby promoting a catalytic cascade with the potential to degrade many components of ECM, such as proteoglycans, collagens, and non-collagenous glycoproteins. For instance, MMP-3 is able to activate pro-MMP-7 (Figure 1B), proMMP-9 (Figure 1C) and pro-MMP-13 (Figure 1D). ${ }^{6}$

In physiological conditions, MMPs are produced at very low level and are involved in the normal tissue turnover. Therefore, production, activation and activity of MMPs are tightly regulated in order to prevent excessive tissue degradation. There are at least 4 levels of regulation: at the level of transcription, at the point of activation from the precursor zymogens, by interaction with specific ECM components, and by TIMP inhibition. There are four human TIMPs knew, TIMP-1, TIMP-2, TIMP-3 and TIMP-4. TIMPs do not inhibit all MMPs at the same level; while TIMP-1 inhibits mostly MMP-1 and MMP3, TIMP-2 suppresses MMP-2 and MMP-9. ${ }^{8,9}$ TIMP-2 acts also as a positive regulator of MMP-2, given its ability to enhance proMMP-2 activation by MMP-14. ${ }^{10}$

TIMPs form with MMPs reversible complexes in a $1: 1$ ratio. $^{11,12}$ Changes in the production of MMPs and/or TIMPs can result in enhanced MMPs activity and damage. The inhibitory effect of TIMPs on MMPs in normal conditions is illustrated in Figure 2A. Within inflammatory microenvironment, MMP activity cannot be longer controlled by TIMPs as a result of at least 3 different situations: overexpression of MMPs can occur without a parallel increase of TIMPs (Figure 2B); MMP concentration may increase minimally, but this can associate with a sudden decrease in the expression of TIMPs (Figure 2C); increased MMP concentration that is accompanied by concomitant decrease in TIMP levels (Figure 2D).
In this review, we summarized the available data about expression and potential role of MMPs in IBD. In particular, we considered studies investigating gene and protein MMP expression as well as MMP activity in mucosal and blood samples of IBD patients. The reader is direct towards other reviews for data regarding the role of other proteases in IBD and the expression and function of MMPs in murine models of colitis.

\section{Mucosal and Circulating Expression of Metalloproteinases in IBD}

A pioneering study by Saarialho-Kere and colleagues documented abundant expression of MMP-1 and MMP-3 RNA in the gastrointestinal tissue around ulcers, including those present in the gut of IBD patients. ${ }^{13}$ Later on, the same group showed that MMPs are made by different cell types in the gut. Specifically, laminin-5-positive and Ki67-negative enterocytes bordering the ulcerations expressed MMP-10 mRNA, macrophages in the vicinity of shedding mucosal epithelium and beneath the necrotic surface of the ulcers were positive for MMP-12 and fibroblastlike cells in such ulcerations were a source of MMP-13. ${ }^{14}$ According to that, Dobre et al documented increased RNA MMP-10 expression in inflamed tissue of UC patients. ${ }^{15}$

Subsequent studies confirmed the greater RNA expression of multiple MMPs in inflamed tissue of both patients with CD and patients with UC as compared to normal and disease (eg, diverticular disease) controls (Table 1). ${ }^{16}$ In the pediatric population, MMP-7 expression was more pronounced in active $\mathrm{CD}$ than in active $\mathrm{UC}$ raising the possibility MMP-7 could help differentiate CD from UC. ${ }^{17,18}$ Another study in paediatric IBD patients confirmed the increased expression of MMP-7 together with MMP-1, -3 , and -10 in gut epithelial cells and lamina propria mononuclear cells (LPMC) of CD and UC. ${ }^{19}$ Interestingly, MMP-1 levels remained increased in UC patients with endoscopic remission but persistent histological inflammation. ${ }^{20}$ Further work aimed at assessing the cell source of other MMPs in IBD revealed that MMP-1, MMP-3, and MMP-9 were highly produced by mucosal myofibroblasts ${ }^{21,22}$ and were also seen in fistulous tracts, ${ }^{23,24}$ while neutrophils produced MMP-2 and MMP-9. ${ }^{25}$ In contrast, epithelial cells on the edge of ulcers produce mainly MMP-7. ${ }^{26,27}$ This latter finding together with data from studies in the airway epithelium ${ }^{28}$ suggest a role for MMP-7 in re-epithelization after injury. MMP-9 gene and protein expression was particularly increased in patients with extensive UC compared to patients with lesions limited to the left colon or healthy controls. ${ }^{29}$ 


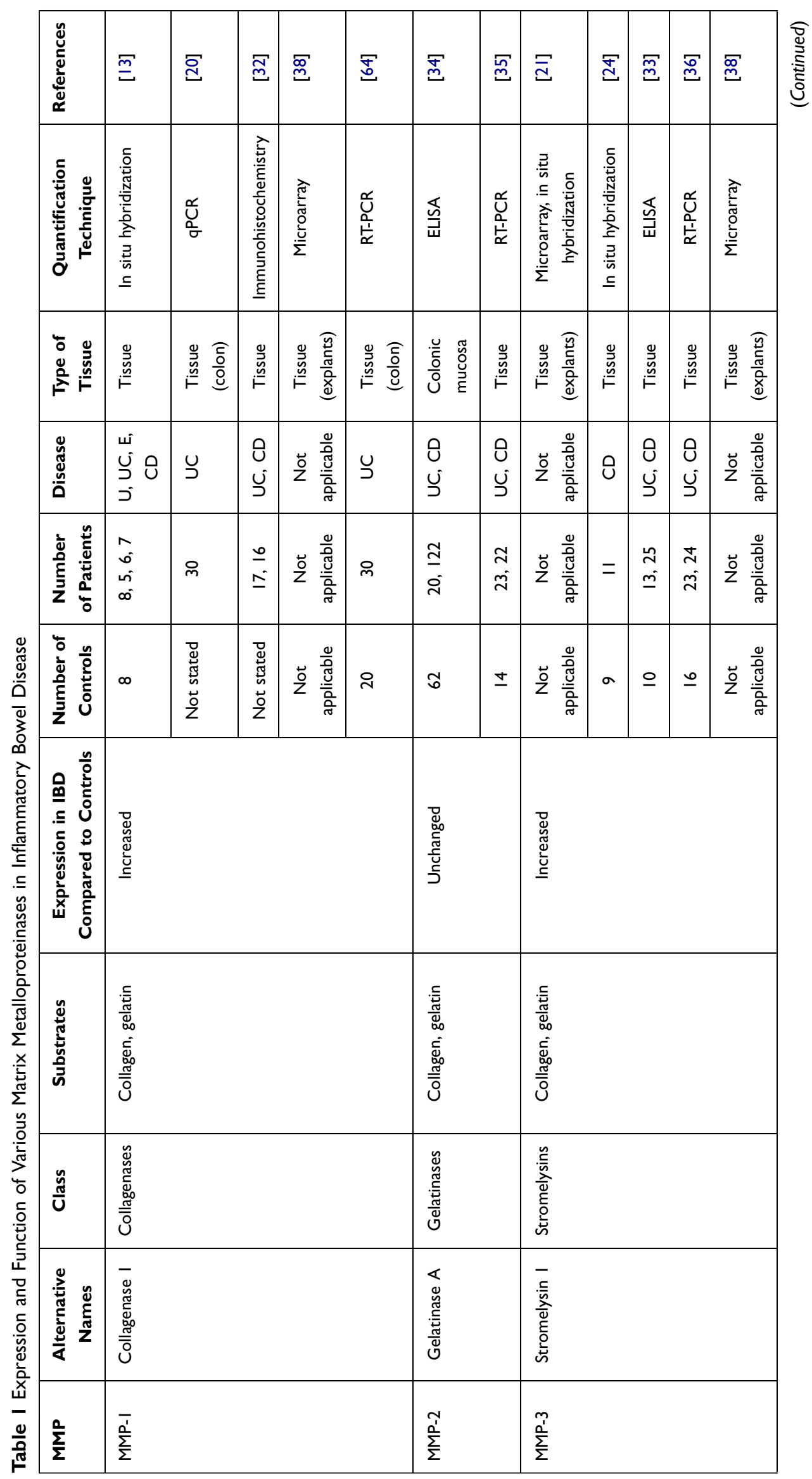




\begin{tabular}{|c|c|c|c|c|c|c|c|c|c|c|c|c|c|}
\hline 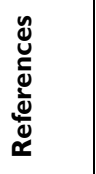 & $\Phi$ & $\stackrel{\Xi}{\check{I}}$ & $\sqrt{\mathrm{d}}$ & 包 & 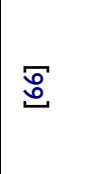 & $\Sigma$ & $\overline{\bar{\Xi}}$ & $\stackrel{\Xi}{\Xi}$ & $\stackrel{\Xi}{d}$ & ఫ్త & $\overrightarrow{\widetilde{n}}$ & $\stackrel{\Phi}{\tilde{n}}$ & 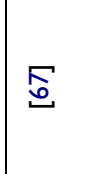 \\
\hline 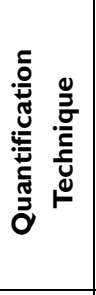 & 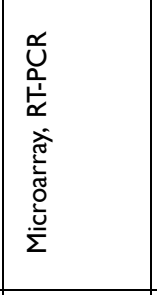 & 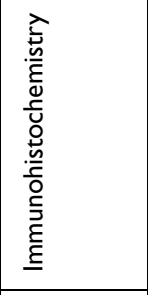 & $\begin{array}{l}\text { U. } \\
\text { 趸 } \\
\frac{1}{\alpha}\end{array}$ & 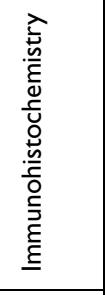 & $\begin{array}{l}\underset{U}{0} \\
\dot{O}\end{array}$ & 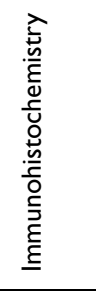 & 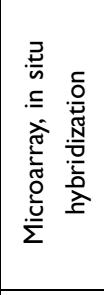 & 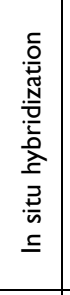 & 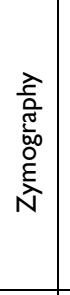 & $\begin{array}{l}\mathscr{U} \\
\stackrel{0}{\sigma}\end{array}$ & 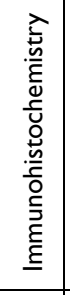 & 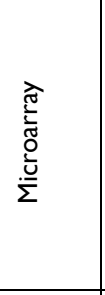 & 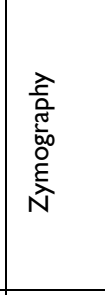 \\
\hline 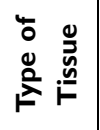 & 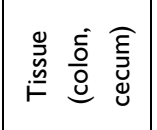 & 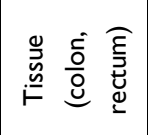 & 总总 & 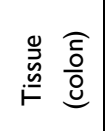 & 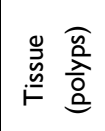 & 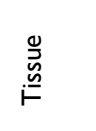 & 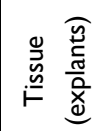 & 总 & 总 & 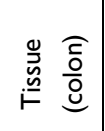 & 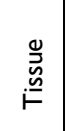 & 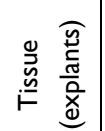 & $\frac{\underline{\underline{u}}}{\tilde{U}}$ \\
\hline 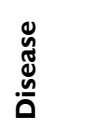 & $\begin{array}{l}\text { Uิ } \\
\text { ù }\end{array}$ & $\mathrm{u}$ & u & $\mathrm{Y}$ & $\begin{array}{l}\dot{\theta}_{0} \\
\text { ù }\end{array}$ & $\begin{array}{l}\dot{U} \\
\dot{u}\end{array}$ & 苍 & 8 & $\begin{array}{l}\mathrm{U} \\
\mathrm{U}\end{array}$ & u & $\begin{array}{l}\mathrm{Q} \\
\mathrm{u} \\
\mathrm{y}\end{array}$ & 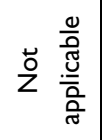 & 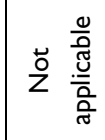 \\
\hline 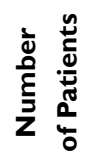 & 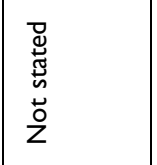 & in & $\stackrel{m}{m}$ & $\stackrel{\sim}{\Lambda}$ & 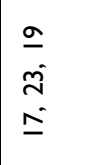 & $\begin{array}{l}= \\
\simeq\end{array}$ & 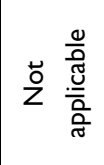 & $=$ & $\begin{array}{l}\frac{m}{\dot{m}} \\
\dot{m}\end{array}$ & $\underline{\circ}$ & $\begin{array}{l}\simeq \\
\simeq\end{array}$ & 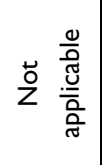 & ž \\
\hline 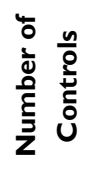 & 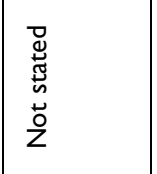 & 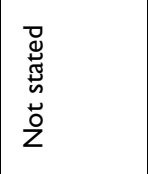 & 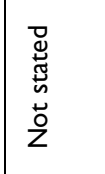 & $\sigma$ & $\underline{\underline{a}}$ & $=$ & 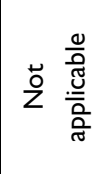 & a & $\sigma$ & $\infty$ & $\begin{array}{l}\stackrel{\mathrm{d}}{\mathrm{J}} \\
\mathrm{w} \\
\mathrm{o} \\
\mathrm{z}\end{array}$ & 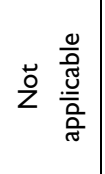 & 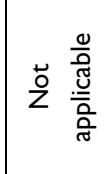 \\
\hline 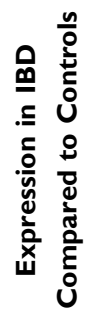 & 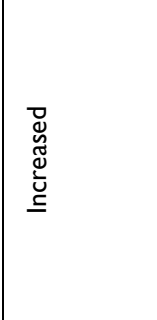 & & & & & 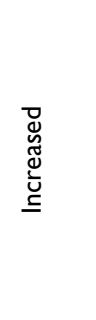 & 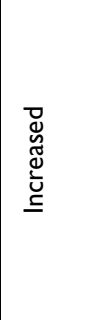 & & & & & & \\
\hline 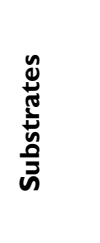 & 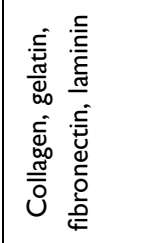 & & & & & 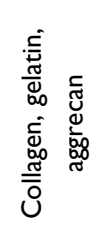 & 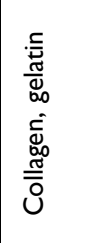 & & & & & & \\
\hline 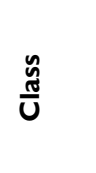 & 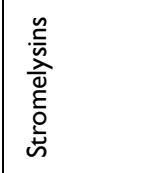 & & & & & 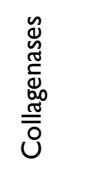 & 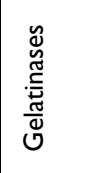 & & & & & & \\
\hline 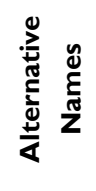 & 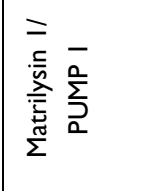 & & & & & 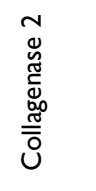 & 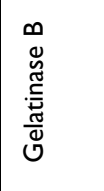 & & & & & & \\
\hline$\frac{0}{\Sigma}$ & 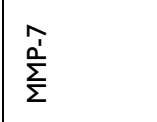 & & & & & 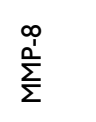 & $\begin{array}{l}\frac{\hat{\nu}}{\sum^{2}} \\
\frac{1}{2}\end{array}$ & & & & & & \\
\hline
\end{tabular}




\begin{tabular}{|c|c|c|c|c|c|c|c|c|c|c|c|c|c|c|}
\hline$\underline{\underline{n}}$ & $\Xi$ & $\underline{\sigma}$ & 怘 & $\overline{\bar{\Sigma}}$ & 买 & $\Xi$ & $\mathbb{\Xi}$ & $\overline{\bar{\Sigma}}$ & 合 & $\overline{\bar{\Sigma}}$ & $\stackrel{\check{n}}{\underline{n}}$ & & & $\overline{\bar{\Delta}}$ \\
\hline 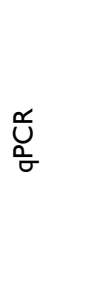 & 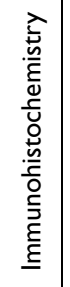 & 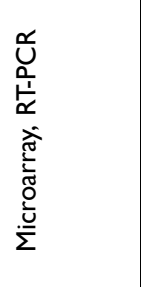 & $\begin{array}{l}\text { Ũ } \\
0 \\
\sigma\end{array}$ & 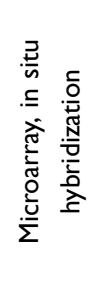 & 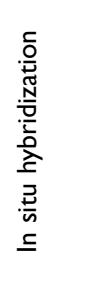 & 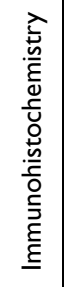 & 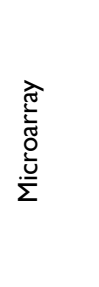 & 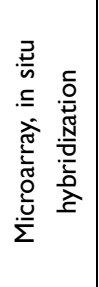 & 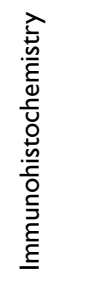 & 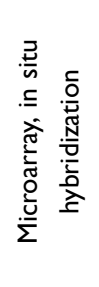 & 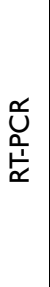 & & & 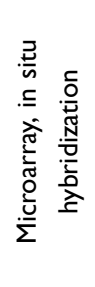 \\
\hline 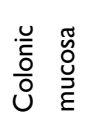 & $\stackrel{\mathscr{o}}{\stackrel{\underline{w}}{\models}}$ & 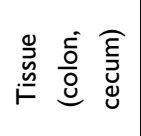 & 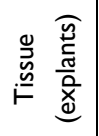 & 竞 & 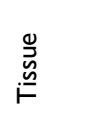 & 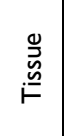 & 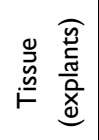 & 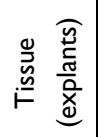 & $\stackrel{0}{\stackrel{0}{\bar{\omega}}}$ & 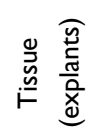 & 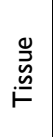 & & & 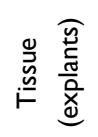 \\
\hline $\begin{array}{l}0 \\
\text { ù } \\
\text { ù }\end{array}$ & $\begin{array}{l}\text { U } \\
\dot{U}\end{array}$ & $\begin{array}{l}\text { Ù } \\
\text { ב̀ }\end{array}$ & $\underline{u}$ & 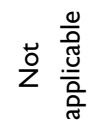 & $\frac{\underline{u}}{\mathrm{u}}$ & $\begin{array}{l}0 \\
u \\
u \\
\text { د }\end{array}$ & $\begin{array}{l}\text { Ù } \\
\text { ù }\end{array}$ & 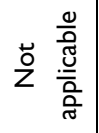 & 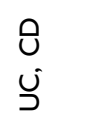 & 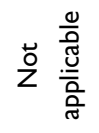 & $\begin{array}{l}\text { Û } \\
\text { ư }\end{array}$ & & & 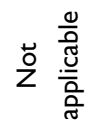 \\
\hline $\begin{array}{l}\tilde{N} \\
\dot{\bar{N}}\end{array}$ & $\begin{array}{l}= \\
\simeq\end{array}$ & 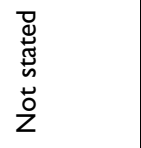 & in & 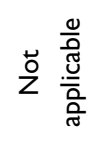 & 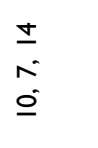 & $\begin{array}{l}= \\
\simeq\end{array}$ & 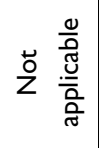 & 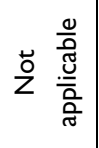 & $\begin{array}{l}\stackrel{ \pm}{2} \\
\text { 峁 }\end{array}$ & 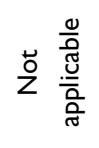 & 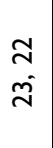 & & & 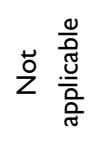 \\
\hline $\bar{N}$ & $=$ & 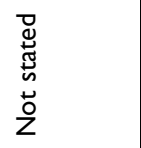 & 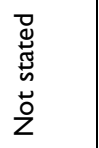 & z) & n & $=$ & 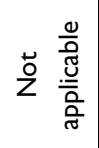 & 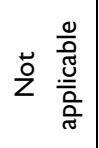 & ஓ & 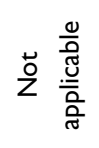 & \pm & & & ž \\
\hline 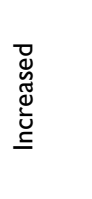 & & & & 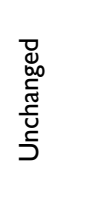 & 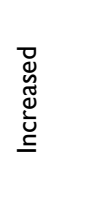 & & & 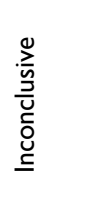 & & 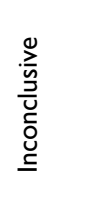 & & 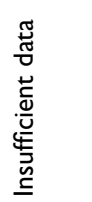 & 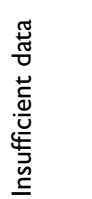 & 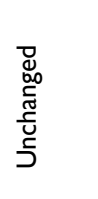 \\
\hline 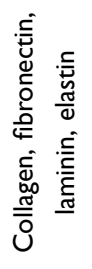 & & & & 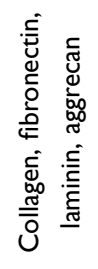 & 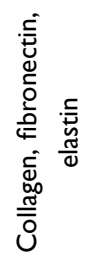 & & & 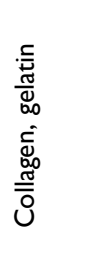 & & 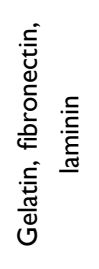 & & 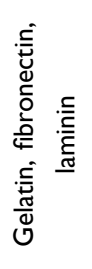 & 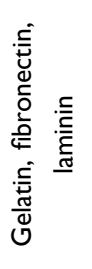 & 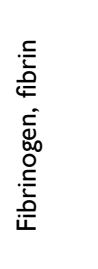 \\
\hline 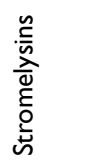 & & & & 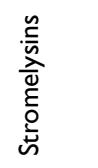 & 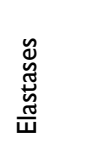 & & & 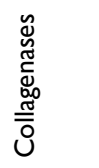 & & 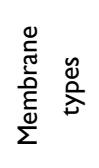 & & 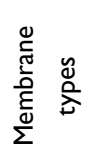 & 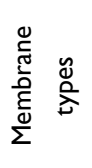 & 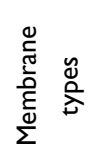 \\
\hline 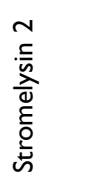 & & & & 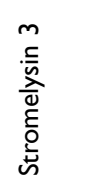 & 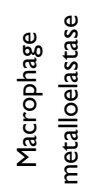 & & & 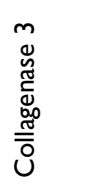 & & 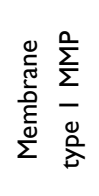 & & 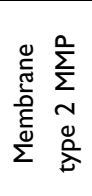 & 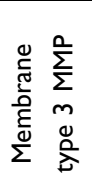 & 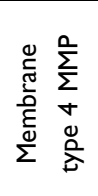 \\
\hline$\frac{O}{\sum_{1}^{1}}$ & & & & $\overline{\overline{\bar{n}}}$ & $\frac{\stackrel{N}{\bar{\Lambda}}}{\frac{\Lambda^{\prime}}{\Sigma}}$ & & & $\frac{\frac{m}{1}}{\frac{a^{\prime}}{\Sigma}}$ & & 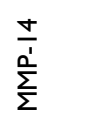 & & $\frac{\frac{n}{1}}{\sum^{\frac{n}{n}}}$ & $\frac{\frac{0}{1}}{\sum_{\Sigma}^{\frac{1}{2}}}$ & $\frac{\bar{n}}{\frac{1}{\Sigma}}$ \\
\hline
\end{tabular}




\begin{tabular}{|c|c|c|c|c|c|c|c|c|c|c|c|}
\hline 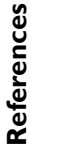 & $\overline{\bar{\beth}}$ & $\Sigma$ & & & $\bar{\Sigma}$ & & & 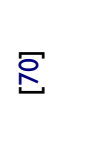 & & 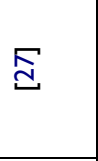 & 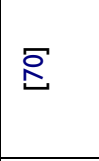 \\
\hline 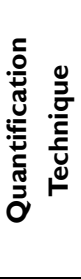 & 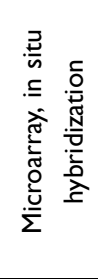 & 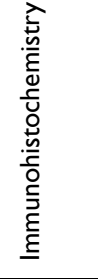 & & & $\begin{array}{l}\text { Ư⿱ 口) } \\
\frac{1}{\alpha x}\end{array}$ & & & 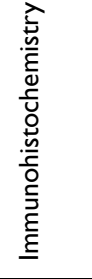 & & $\begin{array}{l}\text { 엄 } \\
\frac{1}{\alpha 1} \\
\frac{1}{\alpha}\end{array}$ & 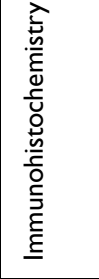 \\
\hline 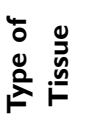 & 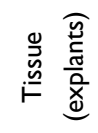 & 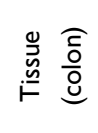 & & & 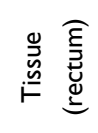 & & & 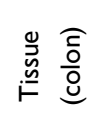 & & 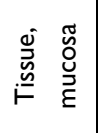 & 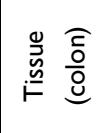 \\
\hline 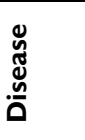 & 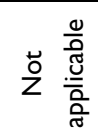 & $\begin{array}{l}\underline{u} \\
\underline{u} \\
\\
\end{array}$ & & & 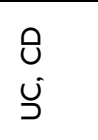 & & & $\begin{array}{l}\underline{u} \\
\underline{u} \\
\\
\end{array}$ & & u & 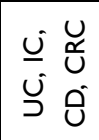 \\
\hline 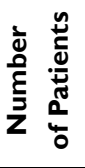 & 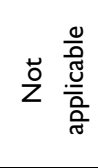 & $\begin{array}{l}\stackrel{\sim}{N} \\
\hat{a} \\
\dot{N}\end{array}$ & & & $\begin{array}{l}0 \\
\dot{m} \\
\dot{q}\end{array}$ & & & $\begin{array}{l}\stackrel{i}{N} \\
\tilde{N} \\
\dot{N} \\
\dot{N}\end{array}$ & & $\stackrel{\llcorner}{m}$ & $\begin{array}{l}\stackrel{i}{1} \\
\hat{\sigma} \\
\dot{j}\end{array}$ \\
\hline 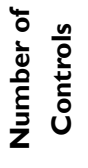 & 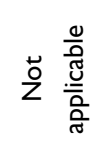 & in & & & 유 & & & in & & 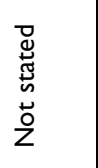 & in \\
\hline 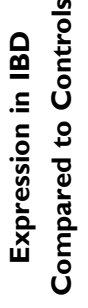 & 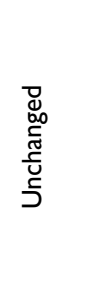 & & 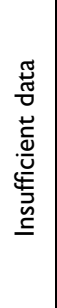 & 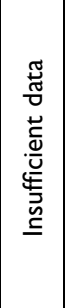 & 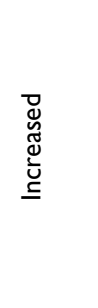 & 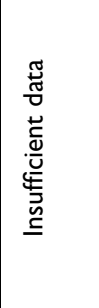 & 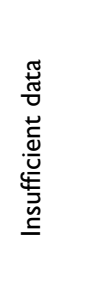 & 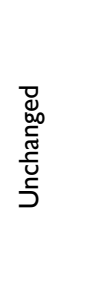 & 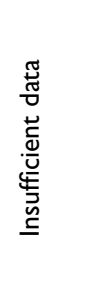 & 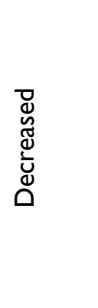 & \\
\hline 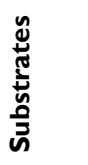 & $\begin{array}{l}\frac{5}{5} \\
0 \\
\frac{5}{5} \\
\text { s. }\end{array}$ & & $\begin{array}{l}\frac{5}{3} \\
0 \\
\frac{5}{5} \\
5\end{array}$ & $\begin{array}{l}\frac{5}{0} \\
\frac{0}{5} \\
\frac{5}{5}\end{array}$ & $\begin{array}{l}\frac{5}{3} \\
0 \\
\frac{5}{5} \\
5\end{array}$ & $\begin{array}{l}\frac{5}{5} \\
0 \\
\frac{5}{5} \\
\text { J }\end{array}$ & $\begin{array}{l}\frac{5}{3} \\
0 \\
\frac{5}{5} \\
5\end{array}$ & $\begin{array}{l}\frac{5}{3} \\
0 \\
\frac{5}{5} \\
\text { s. }\end{array}$ & $\begin{array}{l}\frac{5}{3} \\
0 \\
\frac{5}{5} \\
5\end{array}$ & $\begin{array}{l}\frac{5}{3} \\
0 \\
\frac{5}{5} \\
5\end{array}$ & \\
\hline$\frac{\tilde{\tilde{U}}}{\tilde{U}}$ & 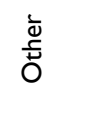 & & $\begin{array}{l}\overline{\bar{\Xi}} \\
\text { ț }\end{array}$ & 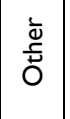 & 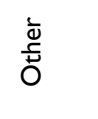 & 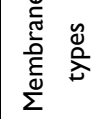 & 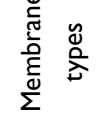 & 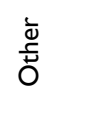 & $\begin{array}{l}\overline{\bar{o}} \\
\text { O̦ }\end{array}$ & 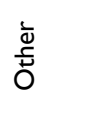 & \\
\hline 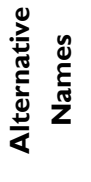 & 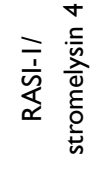 & & 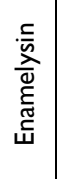 & $\sum_{\sum_{x}^{0}}^{0}$ & $\sum_{\substack{\dot{S} \\
U}}^{0}$ & 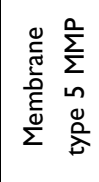 & 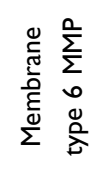 & 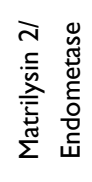 & 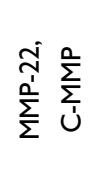 & 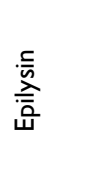 & \\
\hline$\frac{0}{\Sigma}$ & $\frac{\sigma}{\frac{\sigma}{\sum^{\prime}}}$ & & 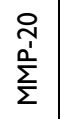 & $\begin{array}{l}\bar{N} \\
\sum_{\Sigma}^{\prime} \\
\bar{\Sigma}\end{array}$ & $\begin{array}{l}\underset{T}{T} \\
\underset{\Sigma}{\Lambda}\end{array}$ & $\begin{array}{l}\stackrel{ \pm}{N} \\
\underset{\sum}{\Delta}\end{array}$ & $\begin{array}{l}\stackrel{\sim}{T} \\
\stackrel{\underline{\Lambda}}{\Sigma} \\
\Sigma\end{array}$ & $\begin{array}{l}\stackrel{0}{T} \\
\sum_{\Sigma}^{\frac{1}{\Sigma}}\end{array}$ & 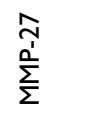 & 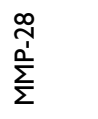 & \\
\hline
\end{tabular}



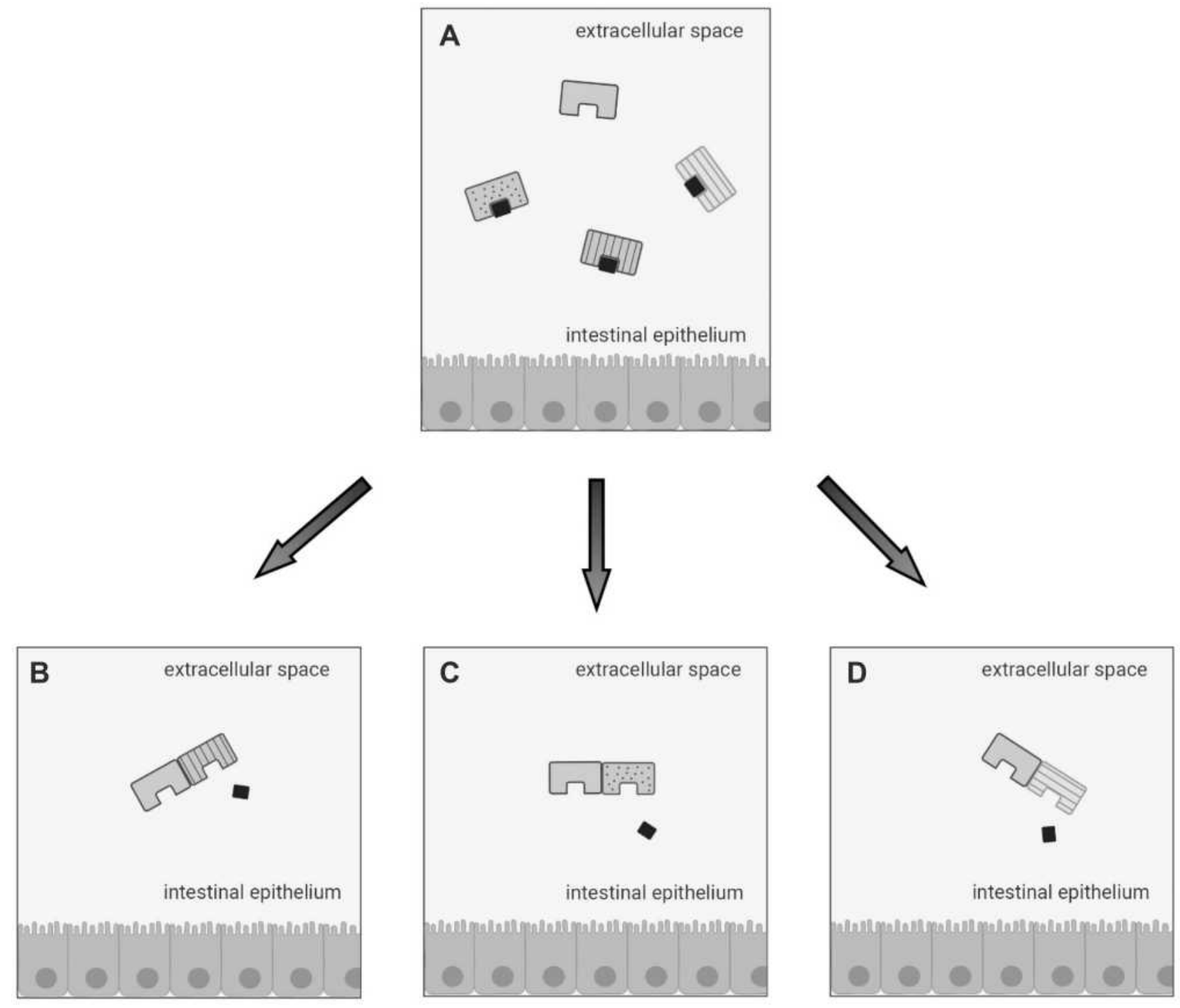

MMP-7 个

MMP-9 $\uparrow$

MMP-13 个
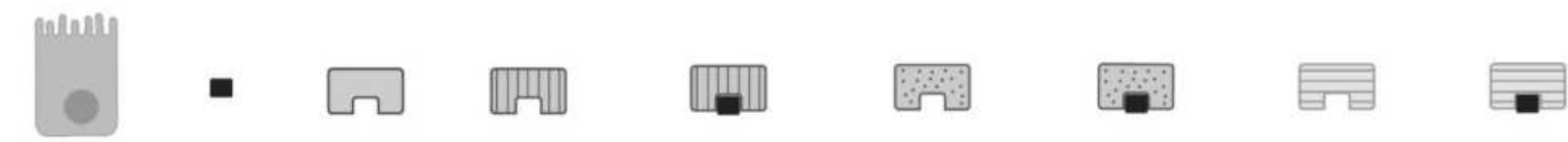

$\begin{array}{cccccccc}\begin{array}{c}\text { intestinal } \\ \text { epithelial domain } \\ \text { cell }\end{array} & \begin{array}{c}\text { pro } \\ \text { d }\end{array} & \begin{array}{c}\text { MMP-3 } \\ \text { (active) }\end{array} & \begin{array}{c}\text { MMP-7 } \\ \text { (inactive, with } \\ \text { pro domain } \\ \text { attached) }\end{array} & \begin{array}{c}\text { MMP-9 } \\ \text { (active) }\end{array} & \begin{array}{c}\text { MMP-9 } \\ \text { (inactive, with } \\ \text { pro domain } \\ \text { attached) }\end{array} & \begin{array}{c}\text { MMP-13 } \\ \text { (active) }\end{array} & \begin{array}{c}\text { MMP-13 } \\ \text { (inactive, with } \\ \text { pro domain } \\ \text { attached) }\end{array}\end{array}$

Figure I Schematic view illustrating how a specific matrix metalloproteinase (MMP) (eg, MMP-3 [A]) triggers the conversion of other MMP zymogens (proMMP-7 [B], proMMP-9 [C], or proMMP-13 [D]) to their active forms. 


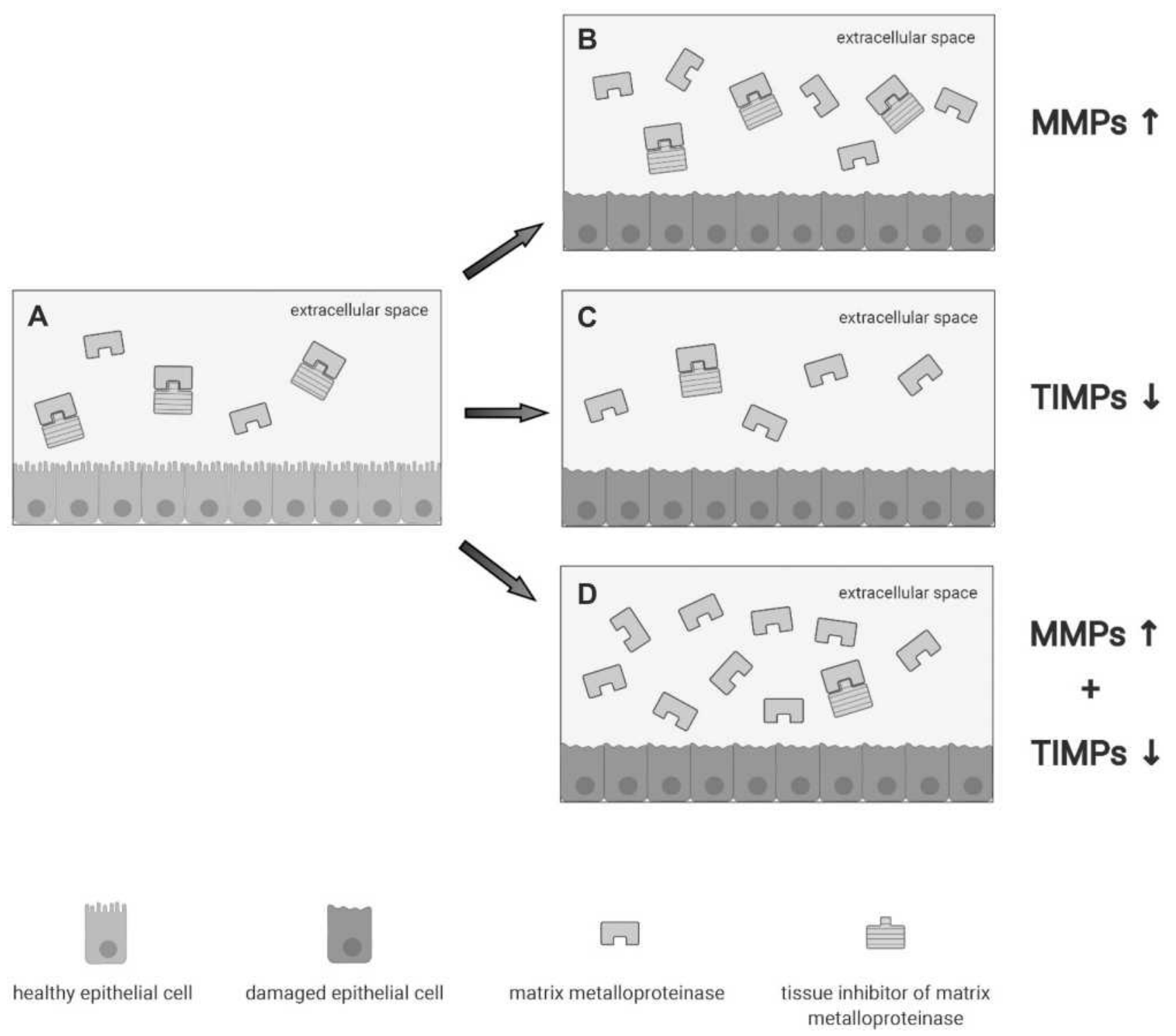

Figure 2 Schematic view of situations in which MMP is either controlled or not by tissue inhibitors of MMPs (TIMPs). (A) In physiological conditions, TIMPs form with MMPs reversible complexes in a I:I ratio thereby controlling MMP activity. Within inflammatory microenvironment, the TIMP/MMP ratio can be decreased by overexpression of MMPs without a parallel increase of TIMPs (B), a sudden decrease in the expression of TIMPs (C) or increased MMP concentration that is accompanied by concomitant decrease in TIMP levels (D).

IBD are also marked by increased plasma concentration of various MMPs, ${ }^{30,31}$ even though there is no clearcut evidence supporting the use of circulating MMP level as an indicator of disease activity.

Altogether, these studies indicate that IBD-related mucosal inflammation associates with enhanced induction of several MMPs.

\section{TIMPs in IBD}

TIMP-1 is abundantly expressed by vascular smooth muscle cells and pericytes in the ulcer bases of IBD patients ${ }^{13,32}$ as well as in inflamed mucosa of both CD patients and UC patients. ${ }^{33}$ Interestingly, such an increase occurs in IBD mucosal samples exhibiting also elevated activity of MMP-1 and MMP-3, clearly indicating the inability of TIMP-1 to suppress 
MMPs function. ${ }^{34}$ Indeed, higher MMP-1/TIMP-1 and MMP-

3/TIMP-1 ratios were seen in both CD and UC compared to controls. ${ }^{35}$ Other studies showed that TIMP-1 levels remained unaltered in biopsy samples of pediatric IBD patients ${ }^{36}$ or documented low expression of TIMP-1 in IBD..$^{17,24,37}$ Similarly, conflicting results have been published about the expression of TIMP-2 in IBD with the majority of studies documenting no change in TIMP-2 content in IBD patients compared to controls ${ }^{21,26,35,38,39}$ and a single study reporting a more pronounced expression of TIMP- 2 in both CD patients and UC patients compared to controls. ${ }^{40}$ TIMP- 3 is abundant in the intestinal epithelium of IBD patients. ${ }^{14,16,41}$

\section{Involvement of MMPs in the Gut Tissue Damage}

In a series of elegant studies exploring the contribution of MMPs in the immune cell-mediated gut damage, Pender and colleagues showed that activation of $\mathrm{T}$ cells in ex vivo organ cultures of human fetal gut with pokeweed mitogen enhanced expression of MMP-1 and MMP-3 while the concentration of TIMP-1 did not change. ${ }^{38}$ Pharmacological blockade of MMP3 prevented the pokeweed mitogen-driven mucosal degradation. Notably, addition of recombinant MMP-3 at nanomolar concentrations (but not MMP-1, MMP-2 or MMP-9) into the organ culture caused tissue destruction thus supporting the pathogenic role of MMPs in such a model of gut damage.

In the fetal gut model, MMPs production was preceded by induction of interferon (IFN)- $\gamma$ and TNF- $\alpha$ and myofibroblasts located just underneath the epithelium were the major sources of MMPs. Blockade of TNF- $\alpha$ with a soluble p55 TNF receptor-human IgG1 fusion protein reduced the levels of MMP-3 in the culture supernatant and inhibited tissue injury. ${ }^{42}$ Along the same line was the demonstration that activation of $\mathrm{T}$ cells in the fetal gut model with IL-12, a master cytokine over-produced in $\mathrm{CD}^{43,44}$ and stimulating IFN- $\gamma$ and TNF- $\alpha,{ }^{45,46}$ induced TNF-driven MMP production and tissue damage. ${ }^{47}$

The lamina propria cells of activated fetal gut explants express also high levels of MMP-12 RNA. ${ }^{21}$

Overall, these data indicate that activation of $\mathrm{T}$ cells and macrophages in the gut leads to production of inflammatory cytokines, which in turn stimulate both immune and nonimmune cells to produce ECM-degrading enzymes. Support to this notion comes from the observation that $\mathrm{T}$ cell-specific cytokines, such as interleukin (IL)-17 and IL-21, which are upregulated in active IBD, ${ }^{48-50}$ induce myofibroblasts to produce MMPs. ${ }^{51-53}$

\section{Role of MMPs in the Nonresponsiveness of Patients with IBD to Anti-TNF Agents}

Agents blocking TNF- $\alpha$ represent one of the major therapeutic options for IBD patients, but not all the commercial compounds exhibit similar efficacy. In particular, infliximab and adalimumab, which are monoclonal IgG1 antibodies, are effective in inducing and maintain remission in both $\mathrm{CD}$ and $\mathrm{UC}$, even though more than one-third of the patients are primarily unresponsive to these drugs or lose the response over time. ${ }^{54}$ In contrast, etanercept, a dimeric $\mathrm{p} 75 \mathrm{TNF}$ receptor-IgG Fc fusion protein, is not beneficial in $\mathrm{CD} .{ }^{55}$ By incubating infliximab, adalimumab and etanercept with mucosal homogenates from IBD patients or activated recombinant human MMP-3 or MMP-12, Biancheri and colleagues showed that MMPs cleaved all the 3 TNF blockers. ${ }^{56}$ However, after MMP degradation, infliximab and adalimumab functioned as $\mathrm{F}$ (ab')2 fragments, whereas cleaved etanercept lost its TNFneutralization ability. These data could, thus, provide the biological explanation of the different effectiveness of the 3 compounds in IBD. Notably, serum samples of IBD patients who did not respond to anti-TNF therapy contain higher levels of MMP3-/MMP12-cleaved endogenous IgG than those measured in responders suggesting that proteolytic degradation occurring in inflamed gut may be a novel mechanism affecting the bioavailability of TNF blockers and contributing to primary nonresponsiveness in IBD. Further experimentation is needed to confirm these data and ascertain whether other MMPs exert similar effects.

\section{Potential Use of MMPs Inhibitors in IBD}

There is no doubt that, in IBD, the excessive immune response associates with abnormal production of several MMPs and altered MMPs/TIMPs ratios. Functional studies support strongly the involvement of MMPs in the IBDrelated mucosal degradation. Indeed, in the last 2 decades, several inhibitors of MMPs have been developed and used to attenuate gut inflammation in animal models of IBD. For instance, administration of the MMP inhibitor, marimastat, to rats with TNBS-colitis reduced clinical and inflammatory scores of colitis. ${ }^{57}$ In the same animal model, similar results were seen following treatment with the MMP inhibitor batimastat. ${ }^{58}$ O'Sullivan and colleagues showed that rectal administration of the dinitrate-barbiturate, a barbituratebased MMP inhibitor incorporating a nitric oxide donor/ 
mimetic group, to rats with dextran-sulfate sodium (DSS) colitis inhibited the induction and activity of MMP-9 and attenuated the ongoing colitis. ${ }^{59}$ These data have paved the way for the development of compounds to use in the clinic. So far, 3 clinical trials with MMP inhibitors have been performed in IBD. A Phase 1 trial investigating the safety and efficacy of GS-5745 (andecaliximab), a fully humanized high-affinity IgG4 monoclonal antibody that selectively binds and inhibits MMP-9, in moderately-to-severe active UC showed promising results. ${ }^{60}$ Seventy-four patients with UC were randomized to receive single or multiple ascending intravenous $(0.3,1.0,2.5$ or $5.0 \mathrm{mg} / \mathrm{kg})$ every 2 weeks (three total infusions) or 5 weekly subcutaneous doses $(150 \mathrm{mg})$ of GS-5745 or placebo. Overall, the drug was safe, and clinical response occurred in $43 \%$ of the patients receiving GS-5745 compared with $13 \%$ of patients receiving placebo. However, these encouraging results were not confirmed by a Phase $2 / 3$ trial, in which eight weeks of induction treatment with GS-5745 induced rates of clinical response or remission similar to those seen in the placebo group. ${ }^{61}$ GS-5745 has also been tested in a phase 2 trial in patients with moderate-to-severe $\mathrm{CD}$. Unfortunately, 8 weeks of induction treatment did not lead to symptomatic or endoscopic response. ${ }^{62}$ Taken together these findings would seem to indicate that MMP-9 does not play a pathological role in IBD. However, in this context, it is noteworthy GS-5745 has higher affinity for proMMP-9 than active MMP- $9^{63}$ and therefore it might inhibit only partially MMP-2 in the gut. This is supported by the demonstration that, in the phase 1 study, patients with a clinical response to GS-5745 had only partial reductions in MMP-9 tissue levels. Since some MMPs have redundant functions and many of them are activated simultaneously during the active phases of the disease, it remains also plausible that blockade of a single MMP (eg, with GS-5745) can have no impact on the mucosal damage.

\section{Conclusions}

In this review, we summarized the available evidence on the expression and potential role of MMPs in IBD. The majority of the presented studies indicate that IBD-related inflammation is associated with increased expression of MMPs and a decreased capacity of TIMPs to inhibit their action. Furthermore, circumstantial evidence suggests that MMP induction relies on the activity of various inflammatory cytokines, which are over-produced in IBD tissue. Interestingly, some studies suggested that MMP-3 and MMP-12 activity may affect the bioavailability of anti-
TNF agents and be responsible for non-responsiveness to these drugs in some patients. Despite the strong evidence implicating MMPs in IBD pathogenesis, clinical trials using MMPs inhibitors showed disappointing results. Further clinical studies are, however, needed to ascertain whether such drugs can be helpful in specific subgroup of patients (eg, fistulizing/fibrostricturing CD).

\section{Acknowledgment}

Figures were created with BioRender.com.

\section{Author Contributions}

All authors made a significant contribution to the work reported, whether that is in the conception, study design, execution, acquisition of data, analysis and interpretation, or in all these areas; took part in drafting, revising or critically reviewing the article; gave final approval of the version to be published; have agreed on the journal to which the article has been submitted; and agree to be accountable for all aspects of the work.

\section{Funding}

This work was supported by the Slovak Research and Development Agency under the contract no. APVV-170505.

\section{Disclosure}

The authors declared no conflicts of interest in this work.

\section{References}

1. Seyedian SS, Nokhostin F, Malamir MD. A review of the diagnosis, prevention, and treatment methods of inflammatory bowel disease. $J$ Med Life. 2019;12(2):113-122. doi:10.25122/jml-2018-0075

2. MacDonald TT, Monteleone G. Immunity, inflammation, and allergy in the gut. Science. 2005;307(5717):1920-1925. doi:10.1126/ science. 1106442

3. Yang C, Singh P, Singh H, Le M-L, El-Matary W. Systematic review: thalidomide and thalidomide analogues for treatment of inflammatory bowel disease. Aliment Pharmacol Ther. 2015;41(11):1079-1093. doi:10.1111/apt.13181

4. Baldassano RN, Piccoli DA. Inflammatory bowel disease in pediatric and adolescent patients. Gastroenterol Clin North Am. 1999;28 (2):445-458. doi:10.1016/S0889-8553(05)70064-9

5. Dalal SR, Cohen RD. What to Do When Biologic Agents Are Not Working in Inflammatory Bowel Disease Patients. Gastroenterol Hepatol. 2015;11(10):657-665.

6. Biancheri P, Di Sabatino A, Corazza GR, MacDonald TT. Proteases and the gut barrier. Cell Tissue Res. 2013;351(2):269-280. doi:10.1007/s00441-012-1390-z

7. Kucukguven A, Khalil R. Matrix metalloproteinases as potential targets in the venous dilation associated with varicose veins. Curr Drug Targets. 2013;14(3):287-324. 
8. Gomis-Rüth FX, Maskos K, Betz M, et al. Mechanism of inhibition of the human matrix metalloproteinase stromelysin-1 by TIMP-1. Nature. 1997;389(6646):77-81. doi:10.1038/37995

9. Nagase H, Visse R, Murphy G. Structure and function of matrix metalloproteinases and TIMPs. Cardiovasc Res. 2006;69 (3):562-573. doi:10.1016/j.cardiores.2005.12.002

10. Strongin AY, Collier I, Bannikov G, Marmer BL, Grant GA, Goldberg GI. Mechanism of cell surface activation of $72-\mathrm{kDa}$ type IV collagenase. Isolation of the activated form of the membrane metalloprotease. $J$ Biol Chem. 1995;270(10):5331-5338. doi:10.1074/jbc.270.10.5331

11. Gomez D, Alonso D, Yoshiji H, Thorgeirsson U. Tissue inhibitors of metalloproteinases: structure, regulation and biological functions. Eur J Cell Biol. 1997;74(2):111-122.

12. Murphy G, Willenbrock F. Tissue inhibitors of matrix metalloendopeptidases. Methods Enzymol. 1995;248(C):496-510. doi:10.1016/0076-6879(95)48032-3

13. Saarialho-Kere U, Vaalamo M, Puolakkainen P, Airola K, Parks WC, Karjalainen-Lindsberg ML. Enhanced expression of matrilysin, collagenase, and stromelysin-1 in gastrointestinal ulcers. Am J Pathol. 1996;148(2):519-526.

14. Vaalamo M, Karjalainen-Lindsberg ML, Puolakkainen P, Kere J, Saarialho-Kere U. Distinct expression profiles of stromelysin-2 (MMP-10), collagenase-3 (MMP-13), macrophage metalloelastase (MMP-12), and tissue inhibitor of metalloproteinases-3 (TIMP-3) in intestinal ulcerations. Am J Pathol. 1998;152(4):1005-1014.

15. Dobre M, Milanesi E, Mănuc TE, et al. Differential intestinal mucosa transcriptomic biomarkers for Crohn's disease and ulcerative colitis. J Immunol Res. 2018;2018:9208274. doi:10.1155/2018/9208274

16. Altadill A, Eiró N, González LO, et al. Comparative analysis of the expression of metalloproteases and their inhibitors in resected crohn's disease and complicated diverticular disease. Inflamm Bowel Dis. 2012;18(1):120-130. doi:10.1002/ibd.21682

17. Mäkitalo L, Kolho KL, Karikoski R, Anthoni H, Saarialho-Kere U. Expression profiles of matrix metalloproteinases and their inhibitors in colonic inflammation related to pediatric inflammatory bowel disease. Scand J Gastroenterol. 2010;45(7-8):862-871. doi:10.3109/00365520903583863

18. Mäkitalo L, Piekkala M, Ashorn M, et al. Matrix metalloproteinases in the restorative proctocolectomy pouch of pediatric ulcerative colitis. World J Gastroenterol. 2012;18(30):4028-4036. doi:10.3748/wjg.v18.i30.4028

19. Jimbo K, Ohtsuka Y, Kojima Y, et al. Increased expression of CXCR3 axis components and matrix metalloproteinase in pediatric inflammatory bowel disease patients. Pediatr Int. 2014;56 (6):873-883. doi:10.1111/ped.12362

20. Manuc M, Ionescu EM, Milanesi E, et al. Molecular signature of persistent histological inflammation in ulcerative colitis with mucosal healing. J Gastrointest Liver Dis. 2020;29(2):159-166. doi:10.15403/ jgld-576

21. Salmela MT, MacDonald TT, Black D, et al. Upregulation of matrix metalloproteinases in a model of $\mathrm{T}$ cell mediated tissue injury in the gut: analysis by gene array and in situ hybridisation. Gut. 2002;51 (4):540-547. doi:10.1136/gut.51.4.540

22. Pender SLF, Li CKF, Sabatino ADI, Macdonald TT, Buckley MG. Role of macrophage metalloelastase in gut inflammation. In: Annals of the New York Academy of Sciences. Vol. 1072. Blackwell Publishing Inc;2006:386-388. doi:10.1196/annals.1326.019

23. Schuppan D, Freitag T. Fistulising Crohn's disease: MMPs gone awry. Gut. 2004;53(5):622-624. doi:10.1136/gut.2003.034207

24. Kirkegaard T, Hansen A, Bruun E, Brynskov J. Expression and localisation of matrix metalloproteinases and their natural inhibitors in fistulae of patients with Crohn's disease. Gut. 2004;53(5):701-709. doi:10.1136/gut.2003.017442
25. Baugh MD, Perry MJ, Hollander AP, et al. Matrix metalloproteinase levels are elevated in inflammatory bowel disease. Gastroenterology. 1999;117(4):814-822. doi:10.1016/S0016-5085(99)70339-2

26. Matsuno K, Adachi Y, Yamamoto H, et al. The expression of matrix metalloproteinase matrilysin indicates the degree of inflammation in ulcerative colitis. J Gastroenterol. 2003;38(4):348-354. doi:10.1007/ s005350300062

27. Rath T, Roderfeld M, Halwe JM, Tschuschner A, Roeb E, Graf J. Cellular sources of MMP-7, MMP-13 and MMP-28 in ulcerative colitis. Scand J Gastroenterol. 2010;45(10):1186-1196. doi:10.3109/00365521.2010.499961

28. Dunsmore SE, Saarialho-Kere UK, Roby JD, et al. Matrilysin expression and function in airway epithelium. J Clin Invest. 1998;102 (7):1321-1331. doi:10.1172/JCI1516

29. Lin X, Li J, Zhao Q, Feng J-R, Gao Q, Nie J-Y. WGCNA Reveals Key Roles of IL8 and MMP-9 in Progression of Involvement Area in Colon of Patients with Ulcerative Colitis. Curr Med Sci. 2018;38 (2):252-258. doi:10.1007/s11596-018-1873-6

30. Wiercinska-Drapalo A, Jaroszewicz J, Flisiak R, Prokopowicz D. Plasma matrix metalloproteinase-1 and tissue inhibitor of metalloproteinase-1 as biomarkers of ulcerative colitis activity. World J Gastroenterol. 2003;9(12):2843-2845. doi:10.3748/wjg.v9. i12.2843

31. De WY, Tan XY, Zhang K. Correlation of plasma MMP-1 and TIMP-1 levels and the colonic mucosa expressions in patients with ulcerative colitis. Mediators Inflamm. 2009;2009:275072. doi:10.1155/2009/275072

32. Arihiro S, Ohtani H, Hiwatashi N, Torii A, Sorsa T, Nagura H. Vascular smooth muscle cells and pericytes express MMP-1, MMP-9, TIMP-1 and type I procollagen in inflammatory bowel disease. Histopathology. 2001;39(1):50-59. doi:10.1046/j.13652559.2001.01142.x

33. Louis E, Ribbens C, Godon A, et al. Increased production of matrix metalloproteinase- 3 and tissue inhibitor of metalloproteinase-1 by inflamed mucosa in inflammatory bowel disease. Clin Exp Immunol. 2000;120(2):241-246. doi:10.1046/j.13652249.2000.01227.x

34. Meijer MJW, Mieremet-Ooms MAC, van der Zon AM, et al. Increased mucosal matrix metalloproteinase- $1,-2,-3$ and -9 activity in patients with inflammatory bowel disease and the relation with Crohn's disease phenotype. Dig Liver Dis. 2007;39(8):733-739. doi:10.1016/j.dld.2007.05.010

35. Von Lampe B, Barthel B, Coupland SE, Riecken EO, Rosewicz S. Differential expression of matrix metalloproteinases and their tissue inhibitors in colon mucosa of patients with inflammatory bowel disease. Gut. 2000;47(1):63-73. doi:10.1136/gut.47.1.63

36. Heuschkel RB, MacDonald TT, Monteleone G, Bajaj-Elliott M, Smith JAW, Pender SLF. Imbalance of stromelysin-1 and TIMP-1 in the mucosal lesions of children with inflammatory bowel disease. Gut. 2000;47(1):57-62. doi:10.1136/gut.47.1.57

37. Gordon JN, Pickard KM, Di sabatino A, et al. Matrix metalloproteinase-3 production by gut IgG plasma cells in chronic inflammatory bowel disease. Inflamm Bowel Dis. 2008;14 (2):195-203. doi:10.1002/ibd.20302

38. Pender S, Tickle S, Docherty A, Howie D, Wathen N, MacDonald T. A major role for matrix metalloproteinases in $\mathrm{T}$ cell injury in the gut. J Immunol. 1997;158(4):1582-1590.

39. Pender SLF, Braegger C, Günther U, et al. Matrix metalloproteinases in necrotising enterocolitis. Pediatr Res. 2003;54(2):160-164. doi:10.1203/01.PDR.0000072326.23442.C3

40. Lakatos G, Hritz I, Varga MZ, et al. The impact of matrix metalloproteinases and their tissue inhibitors in inflammatory bowel diseases. Dig Dis. 2012;30(3):289-295. doi:10.1159/000336995 
41. Salmela MT, Karjalainen-Lindsberg ML, Jeskanen L, Saarialho-Kere U. Overexpression of tissue inhibitor of metalloproteinases-3 in intestinal and cutaneous lesions of graft-versus-host disease. Mod Pathol. 2003;16(2):108-114. doi:10.1097/01.MP.0000051681.43441.82

42. Pender SLF, Fell J, Chamow S, Ashkenazi A, MacDonald T. A p55 TNF receptor immunoadhesin prevents $\mathrm{T}$ cell-mediated intestinal injury by inhibiting matrix metalloproteinase production. J Immunol. 1998;160(8):4098-4103.

43. Monteleone G, Biancone L, Marasco R, et al. Interleukin 12 is expressed and actively released by Crohn's disease intestinal lamina propria mononuclear cells. Gastroenterology. 1997;112 (4):1169-1178. doi:10.1016/S0016-5085(97)70128-8

44. Parrello T, Monteleone G, Cucchiara S, et al. Up-Regulation of the IL-12 Receptor $\beta 2$ Chain in Crohn's Disease. J Immunol. 2000;165 (12):7234-7239. doi:10.4049/jimmunol.165.12.7234

45. Monteleone G, Parrello T, Luzza F, Pallone F. Response of human intestinal lamina propria $\mathrm{T}$ lymphocytes to interleukin 12: additive effects of interleukin 15 and 7. Gut. 1998;43(5):620-628. doi:10.1136/gut.43.5.620

46. Pallone F, Monteleone G. Interleukin 12 and Th1 responses in inflammatory bowel disease. Gut. 1998;43(6):735-736. doi:10.1136/ gut.43.6.735

47. Monteleone G, MacDonald TT, Wathen NC, Pallone F, Pender SLF. Enhancing lamina propria Th1 cell responses with interleukin 12 produces severe tissue injury. Gastroenterology. 1999;117 (5):1069-1077. doi:10.1016/S0016-5085(99)70391-4

48. Monteleone G, Monteleone I, Fina D, et al. Interleukin-21 enhances T-helper cell type I signaling and interferon- $\gamma$ production in Crohn's disease. Gastroenterology. 2005;128(3):687-694. doi:10.1053/j. gastro.2004.12.042

49. Sarra M, Pallone F, MacDonald TT, Monteleone G. IL-23/IL-17 axis in IBD. Inflamm Bowel Dis. 2010;16(10):1808-1813. doi:10.1002/ ibd. 21248

50. Sarra M, Monteleone I, Stolfi C, et al. Interferon-gamma-expressing cells are a major source of interleukin-21 in inflammatory bowel diseases. Inflamm Bowel Dis. 2010;16(8):1332-1339. doi:10.1002/ ibd. 21238

51. Monteleone G, Pallone F, MacDonald TT. Interleukin-21: a critical regulator of the balance between effector and regulatory T-cell responses. Trends Immunol. 2008;29(6):290-294. doi:10.1016/j. it.2008.02.008

52. Monteleone G, Caruso R, Fina D, et al. Control of matrix metalloproteinase production in human intestinal fibroblasts by interleukin 21. Gut. 2006;55(12):1774-1780. doi:10.1136/gut.2006.093187

53. Bamba S, Andoh A, Yasui H, Araki Y, Bamba T, Fujiyama Y. Matrix metalloproteinase-3 secretion from human colonic subepithelial myofibroblasts: role of interleukin-17. $J$ Gastroenterol. 2003;38 (6):548-554. doi:10.1007/s00535-002-1101-8

54. Sandborn WJ, Targan SR. Biologic therapy of inflammatory bowel disease. Gastroenterology. 2002;122(6):1592-1608. doi:10.1053/ gast.2002.33426

55. Sandborn WJ, Hanauer SB, Katz S, et al. Etanercept for active Crohn's disease: a randomized, double-blind, placebo-controlled trial. Gastroenterology. 2001;121(5):1088-1094. doi:10.1053/gast.2001.28674

56. Biancheri P, Brezski RJ, Di Sabatino A, et al. Proteolytic cleavage and loss of function of biologic agents that neutralize tumor necrosis factor in the mucosa of patients with inflammatory bowel disease. Gastroenterology. 2015;149(6):1564-1574.e3. doi:10.1053/j. gastro.2015.07.002

57. Sykes AP, Bhogal R, Brampton C, et al. The effect of an inhibitor of matrix metalloproteinases on colonic inflammation in a trinitrobenzenesulphonic acid rat model of inflammatory bowel disease. Aliment Pharmacol Ther. 1999;13(11):1535-1542. doi:10.1046/j.1365-2036.1999.00633.x
58. Di Sebastiano P, Di Mola FF, Artese L, et al. Beneficial effects of Batimastat (BB-94), a matrix metalloproteinase inhibitor, in rat experimental colitis. Digestion. 2001;63(4):234-239. doi:10.1159/ 000051895

59. O'Sullivan S, Wang J, Radomski MW, Gilmer JF, Medina C. Novel barbiturate-nitrate compounds inhibit the upregulation of matrix metalloproteinase-9 gene expression in intestinal inflammation through a CGMP-mediated pathway. Biomolecules. 2020;10(5):808. doi:10.3390/biom 10050808

60. Sandborn WJ, Bhandari BR, Fogel R, et al. Randomised clinical trial: a phase 1, dose-ranging study of the anti-matrix metalloproteinase-9 monoclonal antibody GS-5745 versus placebo for ulcerative colitis. Aliment Pharmacol Ther. 2016;44(2):157-169. doi:10.1111/apt.13653

61. Sandborn WJ, Bhandari BR, Randall C, et al. Andecaliximab [Anti-matrix Metalloproteinase-9] induction therapy for ulcerative colitis: a randomised, double-blind, placebo-controlled, phase $2 / 3$ study in patients with moderate to severe disease. J Crohn Colitis. 2018;12(9):1021-1029. doi:10.1093/ecco-jcc/jjy049

62. Schreiber S, Siegel CA, Friedenberg KA, et al. A phase 2, randomized, placebo-controlled study evaluating matrix metalloproteinase9 inhibitor, andecaliximab, in patients with moderately to severely active Crohn's disease. J Crohn Colitis. 2018;12(9):1014-1020. doi:10.1093/ecco-jcc/jjy070

63. Appleby TC, Greenstein AE, Hung M, et al. Biochemical characterization and structure determination of a potent, selective antibody inhibitor of human MMP9. J Biol Chem. 2017;292(16):6810-6820. doi:10.1074/jbc.M116.760579

64. De WY, Yan PY. Expression of matrix metalloproteinase-1 and tissue inhibitor of metalloproteinase-1 in ulcerative colitis. World J Gastroenterol. 2006;12(37):6050-6053. doi:10.3748/wjg.v12.i37.6050

65. Newell KJ, Matrisian LM, Driman DK. Matrilysin (matrix metalloproteinase-7) expression in ulcerative colitis-related tumorigenesis. Mol Carcinog. 2002;34(2):59-63. doi:10.1002/ mc. 10049

66. Rath T, Roderfeld M, Graf J, et al. Enhanced expression of MMP-7 and MMP-13 in inflammatory bowel disease: a precancerous potential? Inflamm Bowel Dis. 2006;12(11):1025-1035. doi:10.1097/01.mib.0000234133.97594.04

67. Baugh MD, Hollander AP, Evans GS. The regulation of matrix metalloproteinase production in human colonic fibroblasts. In: Annals of the New York Academy of Sciences. Vol. 859. New York Academy of Sciences; 1998:175-179. doi:10.1111/j.1749-6632.1998. tb11121.x

68. Salmela MT, Pender SLF, Karjalainen-Lindsberg ML, Puolakkainen P, MacDonald TT, Saarialho-Kere U. Collagenase-1 (MMP-1), matrilysin-1 (MMP-7), and stromelysin-2 (MMP-10) are expressed by migrating enterocytes during intestinal wound healing. Scand J Gastroenterol. 2004;39(11):1095-1104. doi:10.1080/ 00365520410003470

69. Vizoso FJ, González LO, Corte MD, et al. Collagenase-3 (MMP-13) expression by inflamed mucosa in inflammatory bowel disease. Scand J Gastroenterol. 2006;41(9):1050-1055. doi:10.1080/ 00365520600554667

70. Bister VO, Salmela MT, Karjalainen-Lendsberg ML, et al. Differential expression of three matrix metalloproteinases, MMP-19, MMP-26, and MMP-28, in normal and inflamed intestine and colon cancer. Dig Dis Sci. 2004;49(4):653-661. doi:10.1023/B: DDAS.0000026314.12474.17

71. Fonseca-Camarillo G, Furuzawa-Carballeda J, Martínez-Benitez B, Barreto-Zuñiga R, Yamamoto-Furusho JK. Increased expression of extracellular matrix metalloproteinase inducer (EMMPRIN) and MMP10, MMP23 in inflammatory bowel disease: cross-sectional study. Scand J Immunol. 2021;93(1):e12962. doi:10.1111/sji.12962 


\section{Publish your work in this journal}

The Journal of Inflammation Research is an international, peerreviewed open-access journal that welcomes laboratory and clinical findings on the molecular basis, cell biology and pharmacology of inflammation including original research, reviews, symposium reports, hypothesis formation and commentaries on: acute/chronic inflammation; mediators of inflammation; cellular processes; molecular mechanisms; pharmacology and novel anti-inflammatory drugs; clinical conditions involving inflammation. The manuscript management system is completely online and includes a very quick and fair peerreview system. Visit http://www.dovepress.com/testimonials.php to read real quotes from published authors.

Submit your manuscript here: https://www.dovepress.com/journal-of-inflammation-research-journal 